\title{
Intelligent mobile detection of cracks in concrete utilising an unmanned aerial vehicle
}

\author{
Khattab M. Ali Alheeti ${ }^{1}$, Muzhir Shaban Al-Ani ${ }^{2}$, Abdulkareem Kareem Najem Al-Aloosy ${ }^{1}$, \\ Abdulkareem Alzahrani ${ }^{3}$, Duaa Abdul Sattar Rukan ${ }^{1}$ \\ ${ }^{1}$ Computer Networking Systems Department, College of Computer Sciences and Information Technology, University of Anbar, Ramadi, \\ Iraq \\ ${ }^{2}$ Information Technology Department, College of Science and Technology, University of Human Development, Sulaymaniyah, Iraq \\ ${ }^{3}$ Computer Science and Engineering Department, Faculty of Computer Science and Information Technology, Al Baha University, Al \\ Baha, Saudi Arabia
}

\begin{abstract}
Article Info
\section{Article history:}

Received Mar 21, 2021

Revised Oct 12, 2021

Accepted Dec 27, 2021

\section{Keywords:}

Concrete crack detection Intelligent mobile communications Support vector machine Unmanned aerial vehicle

ABSTRACT

Cracks in constructions may result in negative consequences in terms of expenditure and safety. This in turn highlights the importance of finding ways to detect these cracks easily and effectively. Hence, technological advances play significant role in enabling effective and innovative ideas, such as the use of autonomous drones and artificial intelligence solutions. In this research, we utilise a type of drone called an unmanned aerial vehicle, equipped with a high-speed camera that can capture images of cracks in buildings, and pass the information to the system. We utilised a dataset that has images collected from different Middle East Technical University (METU) campus buildings with various concrete surfaces (with and without cracks). The crack detection approach uses statistical measures and a support vector machine that prevents overfitting, attains a good rate of accuracy, tackles problems in real-time, and can train a model when a small dataset exists. The combination of an unmanned aerial vehicle, artificial intelligence, and digital image processing gives excellent results. Performance metrics reported for seven rounds of experiments showed rates of accuracy in detection ranging from $83.3-100 \%$ (with $100 \%$ achieved in two rounds). This demonstrates the effectiveness of our proposed detection system in detecting cracks in constructions.
\end{abstract}

This is an open access article under the CC BY-SA license.

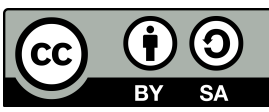

\section{Corresponding Author:}

Abdulkareem Alzahrani

Computer Science and Engineering Department, Faculty of Computer Science and Information Technology

Al Baha University, Alaqiq, 65779-77388, Al Baha, Saudi Arabia

Email: ao.alzahrani@bu.edu.sa

\section{INTRODUCTION}

Fractures or breaks in concrete can lead to separation, which is called a crack. Cracks can occur in many different surfaces, such as buildings, roads, bridges, railway tracks, pavements, automobiles, tunnels, and aircraft. In addition, cracks can be active or dormant. The depth, direction, and width of a dormant crack do not propagate or extend over time, unlike an active crack. There are various types of active crack: longitudinal, miscellaneous, transverse, reflection, and crocodile. There are also various types of dormant crack: micro, sealed, thin, line-like, tiny, mixed, medium, large, minor, and complex crack [1]. Information on cracks, such as length, width, and number of cracks is collected to ensure safety and for structural maintenance. Visible damage of a concrete surface can be measured to evaluate its structural health. Although a visual inspection can detect apparent damage in concrete and is a basic method for detecting cracks, it can be dangerous, even for 
specialists. A visual inspection of a large structure is difficult, as there can be areas that are hard to reach, such as the underside of a bridge [2]. Moreover, the method is not accurate, and it is costly and time-consuming. The cost of repairing and maintaining concrete structures is very high. In addition, there are costs associated with traffic disruption. For these reasons, sensors can be embedded in structures to detect the initiation of corrosion or changes in structural stiffness. Such sensors include optical fibres [3] and vibrating wires [4]. These monitoring systems can detect damage in concrete. Alternatives to visual inspection for crack assessment are digital image processing. The techniques overcome some of the disadvantages and limitations of a visual inspection. Cracks can be detected and recognised automatically by utilising image processing techniques, such as morphological operations, clustering, least squares, gradient descent, histogram equalisation, maximum entropy, particle filter, wavelet transform, and Sobel edge detection. Many companies use unmanned aerial vehicles (UAVs) for visual inspections of concrete structures. UAVs are reliable and easy to use. They can collect a huge amount of data, which can be cumbersome and time-consuming to process. Thus, the time saved in collecting data from a structure is spent on data analysis.

Image processing based on artificial intelligence (AI) can help with detecting cracks. Supervised learning methods are used for image classification, including support vector machines (SVMs), K-nearest neighbours, extreme learning machines, random forests, and AdaBoost. In this paper, a UAV and digital image processing based on an SVM are utilised for a crack detection system. The main contributions of this paper are summarised as shwon in: An UAV-mounted high-speed camera is used to capture images and pass them to processor. A detection system is proposed using a UAV and digital image processing. A SVM is used in the training phase of the detection system.

The proposed system will be applied after the integration of the UAV and digital image processing. SVM will be used to distinguish between samples with and without cracks. The remainder of this paper is organised is being as. Section 2 presents related works. The proposed detection system is described in section 3. Section 4 explains the methodology of this system. The performance measurements are in section 5 . The results of simulation experiments are in section 6. Finally, section 7 presents conclusions and future directions.

\section{RELATED WORKS}

A variety of digital image processing techniques have been proposed for crack detection. In 2015, the authors used a UAV and digital image processing to detect cracks in structures. A simulation verified the accuracy of the proposed method [2]. A prototype of a UAV that utilised image processing techniques was proposed to identify cracks in concrete. To evaluate the proposed system, tests were conducted with walls of different sizes and shapes. The calculated crack widths were similar to the actual widths measured using a crack gauge [5]. Using thresholds, cracks that looked different were assessed. Hence, the results showed that the proposed method is efficient and works, even with degraded images [6]. In addition, a crack identification method used a UAV and hybrid image processing. The authors used an ultrasonic displacement sensor, a camera, and a Wi-Fi module to collect images of cracks. The method successfully measured the width of cracks had collected some information on their length. AI can overcome the limitations in crack detection methods based on image processing [7]. A genetic algorithm was used in a system based on image processing, the percolation model, and genetic programming. The proposed algorithm can effectively and accurately detect cracks in concrete [8]. Furthermore, a method based on a convolutional neural networks (CNNs) was proposed to detect cracks in concrete. Although the proposed algorithm can identify an area with cracks, it did not extract information about the cracks [9]. Zhang et al. [10] used a CNNs to detect cracks in a road. Simulation results showed it had an accuracy of more than $85 \%$, but there were errors in identifying the edges of cracks. Furthermore, random forests were proposed for a novel framework to detect cracks in roads. Simulation results show that the proposed algorithm has a good detection rate [11]. The existing similar works have been summarised in Table 1

Our proposed crack detection system differs from the other works shown in Table 1 as it combines a UAV, digital image processing, and SVM. As the name suggested, SVM capable of advancing the prediction accuracy as well as avoiding data overfitting. In addition, SVM has been applied to tackle various real-time problems such as in hypertext categorisation [12], image classification [13], hand-written recognition [14] face detection [15], object detection [16], fall detection [17], plant species classification [18], credit card fraud classification [19], bioinformatics [20], [21], intrusion detection in smart homes [22], and financial time series forecasting [23]. Hence, these various applications has denoted the effectiveness of SVM, particularly when 
having small training sample [24].

Table 1. Some existing similar works

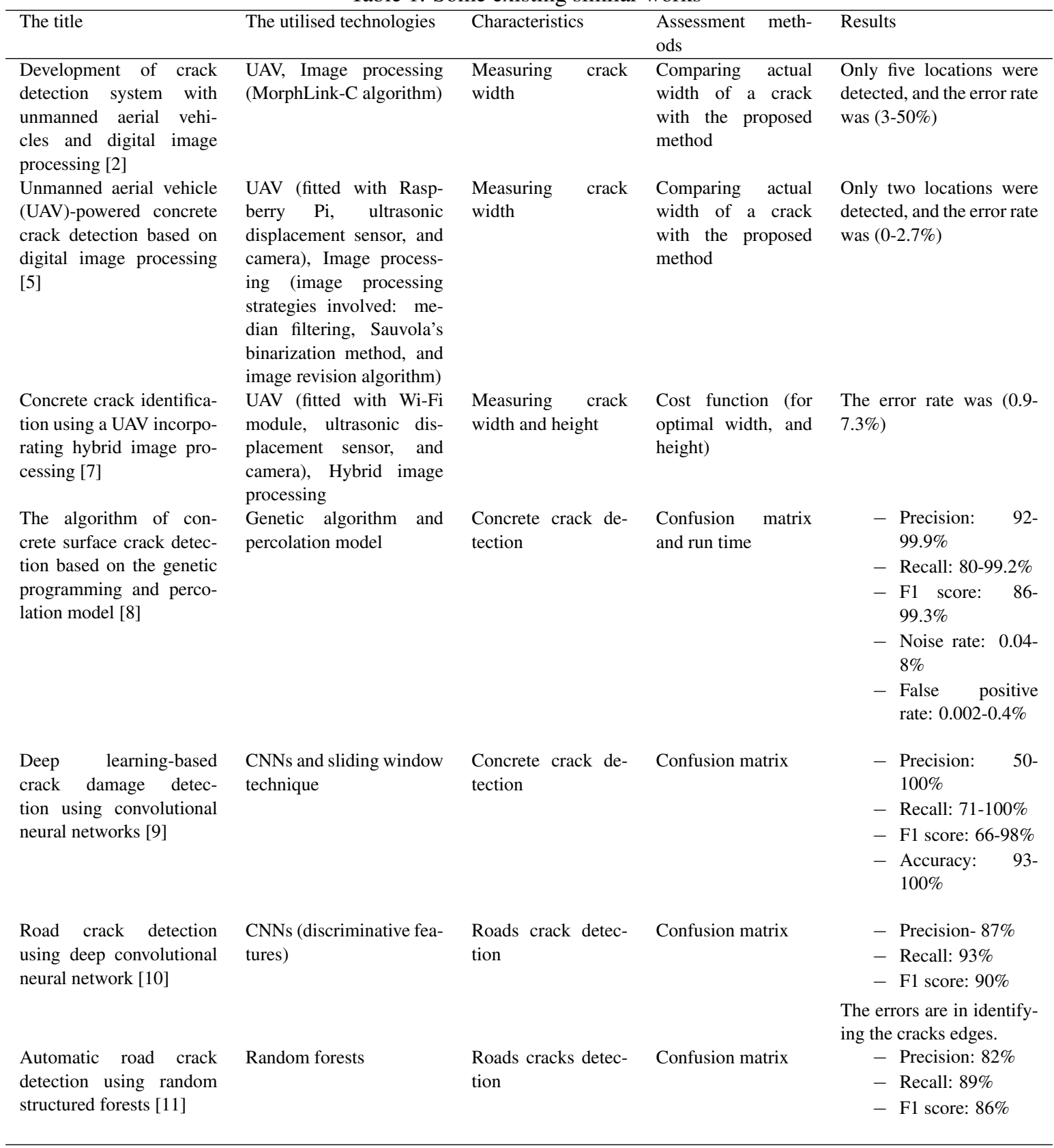

\section{METHODOLOGY OF THE PROPOSED DETECTION SYSTEM}

The proposed system utilises UAV and digital image processing to detect cracks in concrete based on a trained SVM. The steps of the proposed crack detection algorithm are presented as shown in. The life-cycle of the proposed system is illustrated in the algorithm 1 . The algorithm started from collecting images to making decision whether the concrete surface has/has no crack. On the other hand, to build the proposed system, these four main phases should be fulfilled: Collecting an image dataset. Converting the images to numeric values. Training phase. Testing phase. The architecture of the proposed detection system is presented in Figure 1 . Details of each phase are given in the following sections. 

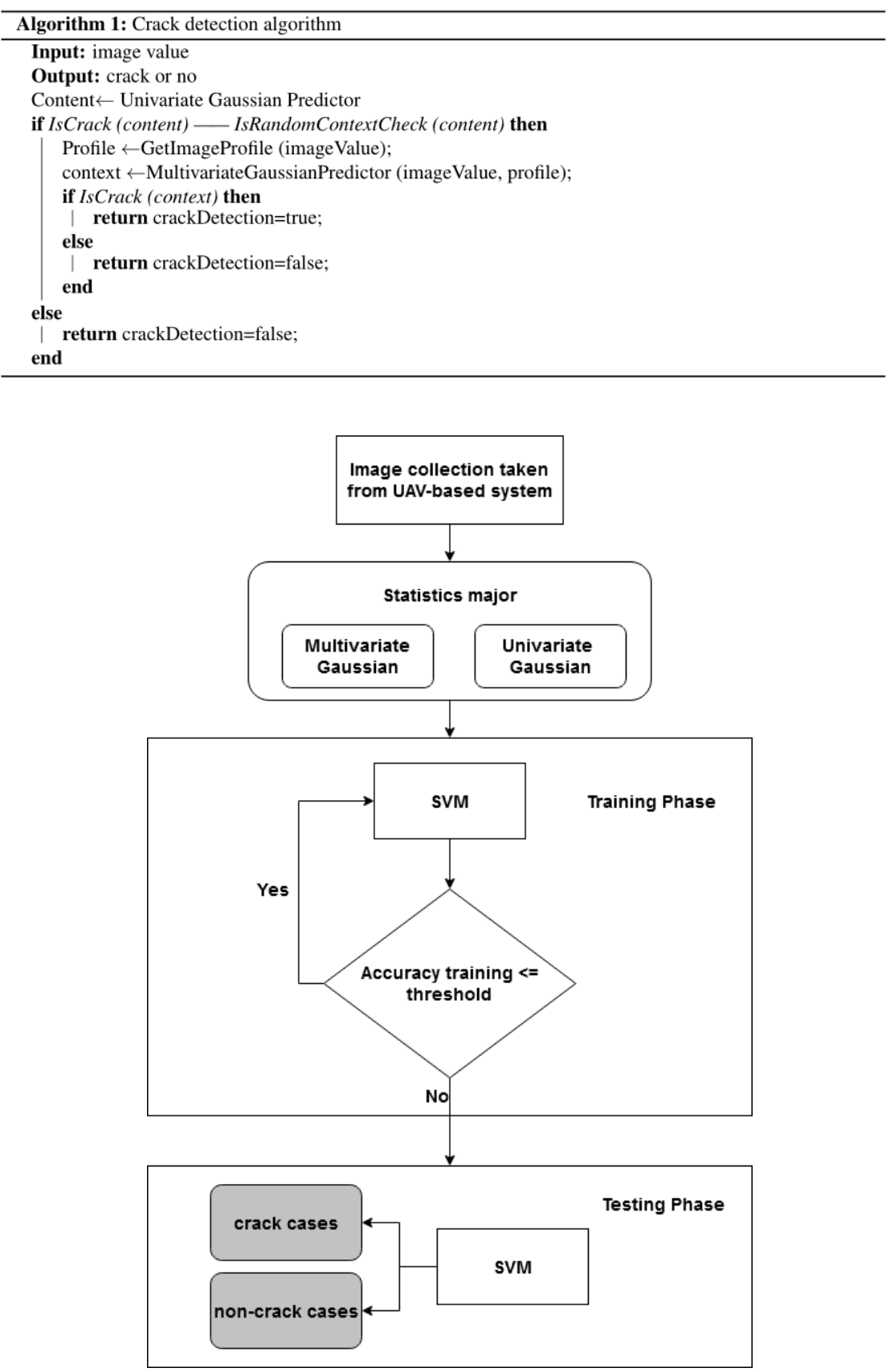

Figure 1. Proposed detection system

\subsection{Image dataset}

The dataset consists of photographs of a variety of different concrete samples from mild cracks to deep and strong cracks. The photographs were captured with an eBee Plus UAV which is provided with a GPS correction system. The latter system applies post-processed kinematic and real-time kinematic technology. The eBee Plus UAV is shown in Figure 2 [25]. The UAV is equipped with a camera designed specifically for drone applications. The camera is fully configurable, ultra-light, dustproof, shockproof, and has 20 megapixel RGB sensor. In addition, the flight plans developed to achieve images with the resolution of $3 \mathrm{~cm}$. For achieving this resolution, the UAV requires to fly $123 \mathrm{~m}$ above the target. The dataset has images of different concrete surfaces with and without cracks (Table 2). They were collected from different METU Campus Buildings. There are 20,000 images of size $227 \times 227$ pixels with RGB channels. The dataset is divided into two folders, one for images classified as positive (with a crack) and the other for negative images (without cracks) [26]. The 
dataset used in this study was divided into three groups: the training set, the validation set, and the test set. Table 1 shows examples of images of concrete surfaces with and without cracks.

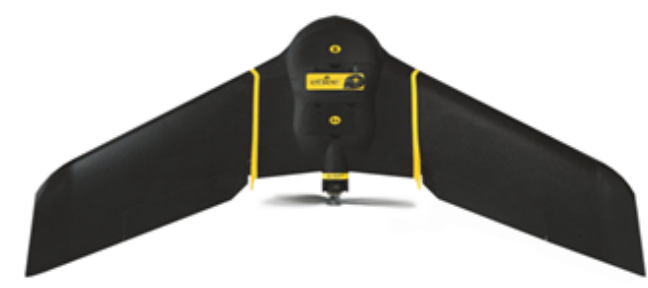

Figure 2. An eBee plus unmanned aerial vehicle

Table 2. Examples of images of concrete surfaces with and without cracks

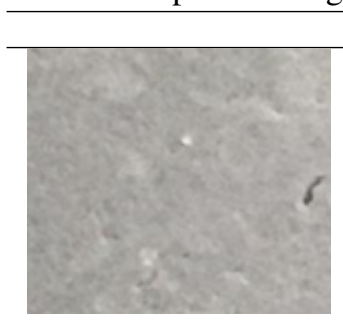

a

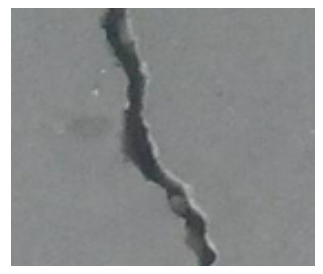

d Images without a crack

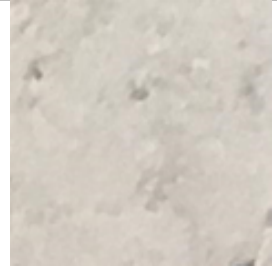

b

Images with a crack

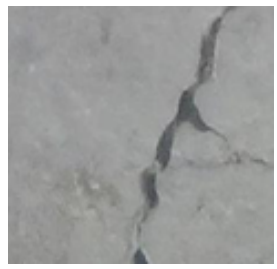

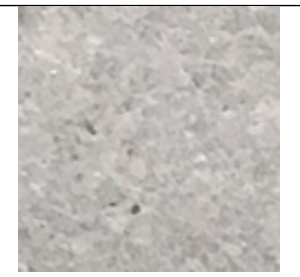

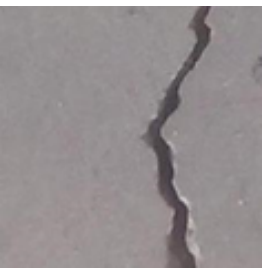

\subsection{Converting image to numeric values}

A statistics major required to transfer the images to numeric values since SVM can work only with numeric values. Most of the advanced sensors (cameras), after the acquisition step, convert the two dimensional signal (image) into digital form using embedded ADC that is suitable to processing.

\subsection{Training phase}

In this research, an SVM is trained as the proposed detection system. An SVM is a classification algorithm based on machine learning concepts. SVM is utilised for processing between the input image and the output images (a classification of the input image). It maximises the accuracy of predictions while avoiding overfitting to the data. SVM is robust, accurate, and effective, even with a small training sample [24]. Therefore, this research aims to employ SVM to improve detection rate and reduce false alarm rate of the proposed detection system. It is worth noting that the threshold was identified to be $99 \%$ in the training phase.

\subsection{Testing phase}

In the testing phase, the confusion matrix, accuracy rate, and error rate were calculated to measure the efficiency of the system. In addition, surveying related works showed that some works did the testing for five positions or less such as in [2] (five locations) and [7]. Hence, we determined to do seven rounds of testing.

\section{PERFORMANCE MEASUREMENT}

In this paper, the efficiency of the proposed system was assessed using performance metrics, such as confusion matrix, the accuracy, and error rate [27]. To calculate the confusion matrix, four alarms were 
calculated:

$$
\begin{aligned}
T P_{\text {Rate(sensitivity) }} & =\frac{T P}{T P+F N} \\
T N_{\text {Rate(specificity })} & =\frac{T N}{T N+F P} \\
F N_{\text {Rate }(1-\text { sensitivity })} & =\frac{F N}{F N+T P} \\
F P_{\text {Rate }(1-\text { specificity })} & =\frac{F P}{F P+T N}
\end{aligned}
$$

Where:

- True positives (TP): the number of images of concrete samples correctly classified as having a crack.

- False positives (FP): the number of images of concrete samples incorrectly classified as having a crack.

- True negatives (TN): the number of images of concrete samples correctly classified as not having a crack.

- False negatives (FN): the number of images of concrete samples incorrectly classified as not having a crack.

In addition, the accuracy rate can be calculated as the ratio of correctly classified patterns to the total number of patterns.

$$
\begin{gathered}
\operatorname{AccuracyRate}(A R)=\frac{T P+T N}{T P+F P+T N+F N} \\
\operatorname{ErrorRate}(E R)=1-\text { Accuracy }
\end{gathered}
$$

\section{EXPERIMENTAL RESULTS}

The confusion matrices, accuracy rates, and error rates are given in Tables 3,9 for rounds 1 to 7 , respectively. As can be shown in the seven tables, the accuracy witness variations amongst the tables $(83.3 \%$ $100 \%$ ) due to the size of the image dataset, and the situation and stability of the round. Hence, the number of iterations can be increased by a larger dataset size to achieve more accurate results. The best accuracy rate for the seven iterations was achieved in the fifth and sixth rounds where the accuracy rate reached $100 \%$. The reason behind this achievement may belong to the reality that these rounds have reached saturation states.

Table 3. Performance metrics of the first round

\begin{tabular}{cccc}
\hline \multicolumn{2}{c}{ Confusion matrix } & Accuracy rate & Error rate \\
\hline TP & $80 \%$ & $99 \%$ & $1 \%$ \\
TN & $100 \%$ & & \\
FN & $20 \%$ & & \\
FP & $0 \%$ & & \\
\hline
\end{tabular}

Table 4. Performance metrics of the second round

\begin{tabular}{cccc}
\hline \multicolumn{2}{l}{ Confusion matrix } & Accuracy rate & Error rate \\
\hline TP & $60 \%$ & $88.5 \%$ & $12.5 \%$ \\
TN & $80 \%$ & & \\
FN & $40 \%$ & & \\
FP & $20 \%$ & & \\
\hline
\end{tabular}

Table 5. Performance metrics of the third round

\begin{tabular}{cccc}
\hline \multicolumn{2}{c}{ Confusion matrix } & Accuracy rate & Error rate \\
\hline TP & $60 \%$ & $88.5 \%$ & $12.5 \%$ \\
TN & $80 \%$ & & \\
FN & $40 \%$ & & \\
FP & $20 \%$ & & \\
\hline
\end{tabular}


Table 6. Performance metrics of the forth round

\begin{tabular}{|c|c|c|c|}
\hline \multicolumn{2}{|c|}{ Confusion matrix } & Accuracy rate & Error rate \\
\hline $\mathrm{TP}$ & $80 \%$ & $100 \%$ & $0 \%$ \\
\hline $\mathrm{TN}$ & $80 \%$ & & \\
\hline FN & $20 \%$ & & \\
\hline FP & $20 \%$ & & \\
\hline
\end{tabular}

Table 7. Performance metrics of the fifth round

\begin{tabular}{cccc}
\hline \multicolumn{2}{c}{ Confusion matrix } & Accuracy rate & Error rate \\
\hline TP & $60 \%$ & $100 \%$ & $0 \%$ \\
TN & $60 \%$ & & \\
FN & $40 \%$ & & \\
FP & $40 \%$ & & \\
\hline
\end{tabular}

Table 8. Performance metrics of the sixth round

\begin{tabular}{cccc}
\hline \multicolumn{2}{c}{ Confusion matrix } & Accuracy rate & Error rate \\
\hline TP & $83.3 \%$ & $91.44 \%$ & $8.33 \%$ \\
TN & $100 \%$ & & \\
FN & $16.7 \%$ & & \\
FP & $0 \%$ & & \\
\hline
\end{tabular}

Table 9. Performance metrics of the seventh round

\begin{tabular}{cccc}
\hline \multicolumn{2}{c}{ Confusion matrix } & Accuracy rate & Error rate \\
\hline TP & $66.6 \%$ & $83.3 \%$ & $16.67 \%$ \\
TN & $100 \%$ & & \\
FN & $33.4 \%$ & & \\
FP & $0 \%$ & & \\
\hline
\end{tabular}

Comparing these outcomes with the related works' outcomes, which was summarized in Table 1 . indicating that an acceptable accuracy rate has been achieved. In addition, Figure 3 compares four experiments [2], [5], [7], [9] with our work in terms of accuracy rates. The comparison shows that we achieved the same high rate of accuracy that was achieved in some previous work (numbers [5], [9]). This in turns indicates the usefulness of our proposed detection method, particularly when the dataset is small, or when avoiding overfitting is intended (thanks to SVM).

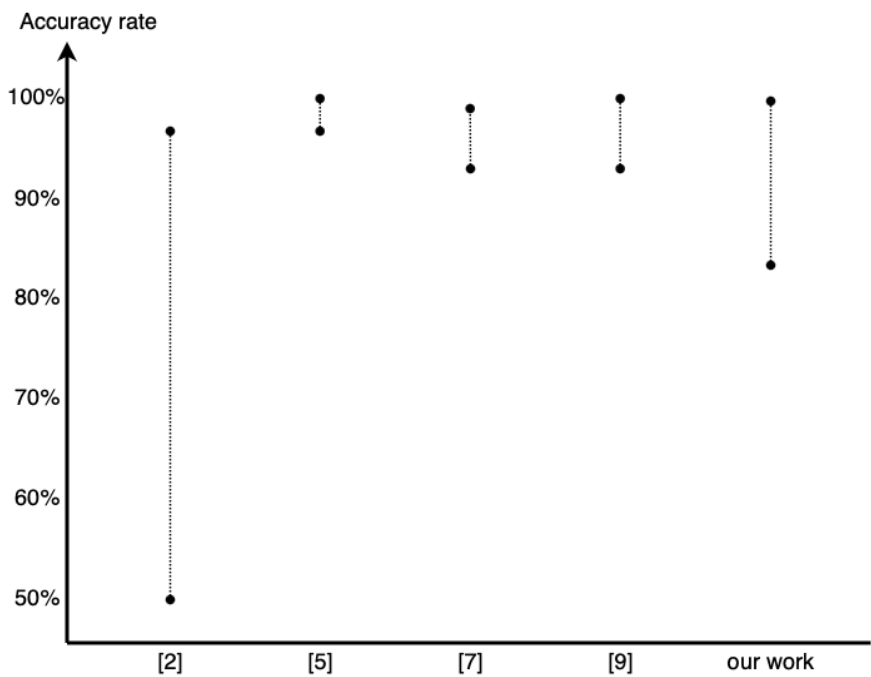

Figure 3. Comparison of accuracy rate between our work and other works 


\section{CONCLUSION AND FUTURE DIRECTION}

Detecting cracks in concrete is a hot topic, which is being studied by many researchers. Various AI and image processing techniques have been utilised to recognise and detect cracks autonomously. Moreover, some researchers have used UAVs to capture images of cracks in concrete structures, and the outcomes have been promising. Therefore, this paper presented a system for detecting cracks in concrete based on integrating a UAV and digital image processing using an SVM. The performance metrics included confusion matrices, accuracy rates, and error rates to verify the performance and efficiency of the proposed detection system. The simulation results showed that the best accuracy rate for seven iterations was $100 \%$ with an error rate of $0 \%$.

In future work, the detection system would be expanded to detect cracks on bridge, pavement, automobile, road, railway track, aircraft, and tunnel. Moreover, the detection system could utilise other AI tools, such as a fuzzy Petri net or linear discrimination analysis. In addition, a better communications system could be integrated into the overall system.

\section{REFERENCES}

[1] S. N. Sitara, "Review and analysis of crack detection and classification techniques based on crack types," International Journal of Applied Engineering Research, vol. 13, no. 8, pp. 6056-6062, 2018, doi: 10.37622/IJAER/13.8.2018.6056-6062.

[2] J.-W. Kim, S.-B. Kim, J.-C. Park, and J.-W. Nam, "Development of crack detection system with unmanned aerial vehicles and digital image processing," Advances in structural engineering and mechanics (ASEM15), vol. 33, no. 3, pp. 25-29, August 2015.

[3] K.-T. Lau, "Fibre-optic sensors and smart composites for concrete applications," Magazine of Concrete Research, vol. 55, no. 1, pp. 19-34, 2003, doi: 10.1680/macr.2003.55.1.19.

[4] A. E. Aktan, et al., "Structural identification for condition assessment: experimental arts," Journal of structural engineering, vol. 123, no. 12 , pp. 1674-1684, 1997.

[5] H. Kim, S.-H. Sim, and S. Cho, "Unmanned aerial vehicle (UAV)-powered concrete crack detection based on digital image processing," in International Conference on Advances in Experimental Structural Engineering, 2015.

[6] E. Aldea and S. Le H'egarat-Mascle, "Robust crack detection for unmanned aerial vehicles inspection in an a-contrario decision framework," Journal of Electronic Imaging, vol. 24, no. 6, p. 061119, 2015, doi: 10.1117/1.JEI.24.6.061119.

[7] H. Kim, J. Lee, E. Ahn, S. Cho, M. Shin, and S.-H. Sim, "Concrete crack identification using a UAV incorporating hybrid image processing," Sensors, vol. 17, no. 9, p. 2052, 2017, doi: 10.3390/s17092052.

[8] Z. Qu, Y.-X. Chen, L. Liu, Y. Xie, and Q. Zhou, "The algorithm of concrete surface crack detection based on the genetic programming and percolation model," in IEEE Access, vol. 7, pp. 57592-57603, 2019, doi: 10.1109/ACCESS.2019.2914259.

[9] Y.-J. Cha, W. Choi, and O. Büyüköztürk, "Deep learning-based crack damage detection using convolutional neural networks," Computer-Aided Civil and Infrastructure Engineering, vol. 32, no. 5, pp. 361- 378, March 2017, doi: 10.1111/mice.12263.

[10] L. Zhang, F. Yang, Y. D. Zhang, and Y. J. Zhu, "Road crack detection using deep convolutional neural network," 2016 IEEE International Conference on Image Processing (ICIP), 2016, pp. 3708-3712, doi: 10.1109/ICIP.2016.7533052.

[11] Y. Shi, L. Cui, Z. Qi, F. Meng, and Z. Chen, ”Automatic road crack detection using random structured forests," in IEEE Transactions on Intelligent Transportation Systems, vol. 17, no. 12, pp. 3434-3445, December 2016, doi: 10.1109/TITS.2016.2552248.

[12] L. H. Lee, C. H. Wan, R. Rajkumar, and D. Isa, "An enhanced support vector machine classification framework by using euclidean distance function for text document categorization," Appl. Intell., vol. 37, no. 1, pp. 80-99, 2012, doi: 10.1007/s10489-011-0314-z.

[13] J. Verma, M. Nath, P. Tripathi, and K. K. Saini, ”Analysis and identification of kidney stone using kth nearest neighbour (KNN) and support vector machine (SVM) classification techniques,"Pattern Recognition and Image Analysis, vol. 27, no. 3, pp. 574-580, 2017, doi: 10.1134/S1054661817030294.

[14] N. A. Jebril, H. R. Al-Zoubi, and Q. Abu Al-Haija, "Recognition of handwritten arabic characters using histograms of oriented gradient (HOG)," Pattern Recognition and Image Analysis , vol. 28, no. 2, pp. 321-345, 2018, doi: 10.1134/S1054661818020141.

[15] Q. Q. Tao, S. Zhan, X. H. Li, and T. Kurihara, "Robust face detection using local CNN and SVM based on kernel combination," Neurocomputing, vol. 211, pp. 98-105, October 2016, doi: 10.1016/j.neucom.2015.10.139.

[16] T. Malisiewicz, A. Gupta, and A. A. Efros, "Ensemble of exemplar-svms for object detection and beyond," 2011 International Conference on Computer Vision, 2011, pp. 89-96, doi: 10.1109/ICCV.2011.6126229.

[17] F. Harrou, N. Zerrouki, Y. Sun, and A. Houacine, "Vision-based fall detection system for improving safety of elderly people," in IEEE Instrumentation Measurement Magazine, vol. 20, no. 6, pp. 49-55, December 2017, doi: 10.1109/MIM.2017.8121952.

[18] J. Cervantes, F. G. Lamont, L. R. Mazahua, A. Z. Hidalgo, and J. S. R. Castilla, "Complex identification of plants from leaves," International Conference on Intelligent Computing. Springer, Cham, Aug. 2018, pp. 376-387, doi: 10.1007/978-3-319-95957-3-41.

[19] J. Jurgovsky, et al., "Sequence classification for credit-card fraud detection," Expert Systems with Applications, vol. 100, pp. 234-245, June 2018, doi: 10.1016/j.eswa.2018.01.037.

[20] L. H. S. Vogado, R. M. S. Veras, F. H. D. Araujo, R. R. V. Silva, and K. R. T. Aires, "Leukemia diagnosis in blood slides using transfer learning in CNNs and SVM for classification," Engineering Applications of Artificial Intelligence, vol. 72, pp. 415-422, June 2018, doi: 10.1016/j.engappai.2018.04.024.

[21] X. Zhang and S. Liu, "RBPPred: predicting RNA-binding proteins from sequence using SVM,” Bioinformatics, vol. 33, no. 6, pp. 854-862, 2017, doi: 10.1093/bioinformatics/btw730.

[22] D. Chowdhry, R. Paranjape, and P. Laforge, "Smart home automation system for intrusion detection," 2015 IEEE 14th Canadian Workshop on Information Theory (CWIT), July 2015, pp. 75-78, doi: 10.1109/CWIT.2015.7255156.

[23] J. Jaramillo, J. D. Velasquez, and C. J. Franco, "Research in financial time series forecasting with svm: Contributions from literature," in IEEE Latin America Transactions, vol. 15, no. 1, pp. 145-153, January 2017, doi: 10.1109/TLA.2017.7827918.

[24] G. Anthony, H. Greg, and M. Tshilidzi, "Classification of images using support vector machines," arXiv:0709.3967, 2007.

[25] e Plus," 2017. [Online]. Available: https://www.sensefly.com/drone/ebee-plus-survey-drone/. 
[26] Ç. F. Özgenel, "Concrete Crack Images for Classification," Mendeley Data, 2019, doi: 10.17632/5y9wdsg2zt.2, [Online]. Available: https://data.mendeley.com/datasets/5y9wdsg2zt/2.

[27] D. Al Dosary, K. M. A. Alheeti, and S. S. Al-Rawi, "An ICMetric Security System for Intelligent Wheelchairs Based on Triple MEMS Sensors," Journal of Southwest Jiaotong University, vol. 54, no. 3, June 2019, doi: 10.35741/issn.0258-2724.54.3.17.

\section{BIOGRAPHIES OF AUTHORS}

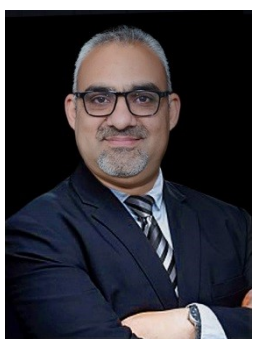

Khattab M. Ali Alheeti (D) 5 P is an Associate Professor at the Department of Computer Networking Systems, College of CS IT - University of Anbar, Iraq, where he has been a faculty member since 2009. From 2018 - now, he was also Head of Computer Networking Systems Deptartment. Alheeti graduated with a first-class honours Msc.Sci. degree in CS IT from AlByte University, Jordan, in 2008, and an PhD. in CS IT from Essex University, UK in 2017. Him research interests are primarily in the area of self-driving vehicles, wireless communications and networks as well as secuity, particularly in neuro-signal processing and intrusion detection, where he is the author/co-author of over 100 research publications. He can be contacted at email: co.khattab.alheeti@uoanbar.edu.iq.

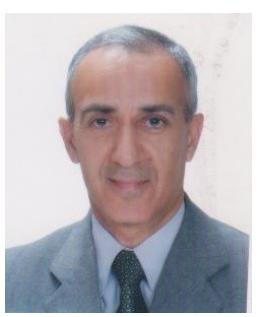

Muzhir Shaban Al-Ani (D) 8 (5) 5 received Ph. D. in Computer Communication Engineering Technology, ETSII School, Spain, Valladolid, 1994. He has more than 40 years of teaching and researching experience and has held several academic positions both inside and outside Iraq. Has 175 published papers and 13 published scientific books. Member and Editor in Chief in a number of international journals. He supervised more than 50 masters and doctoral dissertations. He joined in 1st of October 2016 Department of Information Technology, College of Science and Technology, University of Human Development, Sulaymaniyah, Iraq as a professor. His research of interest concentrated on many field: digital signal, image and medical image processing, IoT and cloud computing, biometric recognition, real time systems, MIS and BIS, speaker identification and human identification. He can be contacted at email: muzhir.al-ani@uhd.edu.iq.

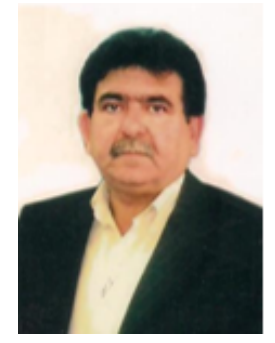

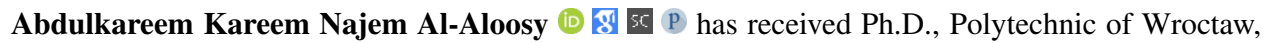
Wroctaw, Poland, 1992. He received training course in UTBM Polytech Engineering School at Marseille - France 2006. He worked 1992-1994 lecturer at the Comp. Engin. Depart., College of Engin., Sabha University-Libya. He worked 1995-1998 in private sector in engin. field. He worked 19992003 Lecturer at the Comp. Eng. Dept. College of engineering/Al Nahrain University-Baghdad. He worked 2004-2006 assistant head of Comp. Eng. Depart., College of Eng./Al Nahrain University-Baghdad. He worked 2006-2009 head of Computer Eng. Depart. in Comp. Man College in Sudan. He worked 2009-2011 associate professor in informatics college in Arab International University in SYRIA. 2011-2015 he joining as head of information system department in Comp. sci. and infor. tech. College/ Anbar University. From 2015-2018 he was the dean of the college, and from 2018 till now he he is a member of the staff in the computer network system department. He can be contacted at email: abdulkareem.alaloosy@uoanbar.edu.iq.

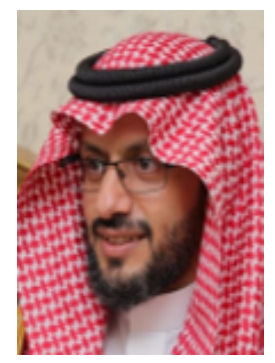

Abdulkareem Alzahrani (1) 18 (5) $P$ received MSc degree in Advanced Web Engineering from the University of Essex, UK (2011) and PhD in Computer Science from the same University (2017). He then works at the Faculty of Computer Science and Information Technology, Al Baha University, Saudi Arabia as an assistant professor. His research interests are: intelligent environments, ambient intelligence, soft computing, and agent/multi-agent systems. He can be contacted at email: ao.alzahrani@bu.edu.sa.

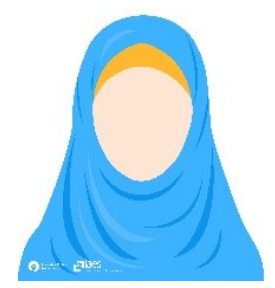

Duaa Abdul Sattar Rukan (i) R: SC P born in 1987, Iraq, Anbar, holds a master's degree in computer science from the College of Computer Science and Information Technology, Anbar University, my interests are in the field of networks and information security. She can be contacted at email: mobileip39@gmail.com. 